\title{
Middle Cerebral Artery Embolectomy After Failed Mechanical Clot Removal
}

\author{
Brian Drake, Gary Redekop
}

Can. J. Neurol. Sci. 2010; 37: 408-411

Intravenous administration of recombinant tissue plasminogen activator (rtPA) has been shown to improve outcomes from ischemic stroke when treatment can be initiated within three hours of symptoms onset. Later administration is associated with a high risk of intracranial hemorrhage. Direct intra-arterial thrombolysis is an option for treatment of selected patients who have a major stroke of less than six hours duration and who are not otherwise candidates for intravenous rtPA. ${ }^{1}$ Recently, endovascular devices have been developed to mechanically extract thrombi from occluded intracranial arteries. The Merci Retriever ${ }^{\circledR}$ (Concentric Medical, Mountain View, CA) has shown promising early results in clinical trials ${ }^{2-4}$ and is recognized as a reasonable intervention for extraction of intraarterial thrombi in carefully selected patients. ${ }^{1}$ We report a case of failed mechanical embolus retrieval in which emergency surgical embolectomy was used to restore cerebral perfusion, leading to an excellent clinical outcome.

\section{Case Report}

\section{Clinical Presentation}

A 51-year-old right-handed male presented to a peripheral hospital with sudden onset of right hemiplegia and aphasia. He was transferred to the Vancouver General Hospital, arriving three hours after symptom onset. His symptoms had improved to a moderate degree but were observed to fluctuate and remained significant. His past medical history was remarkable for a transient ischemic attack four years previously, which presented as an expressive aphasia lasting eight hours, with no definite etiology identified. He was treated with clopidogrel for two years and then the medication was discontinued. He was a cigarette smoker with a 38 pack-year history, and had a history of ongoing alcohol abuse.

On examination his blood pressure was 135/65 and he was in normal sinus rhythm. He was alert but had a moderately severe expressive and receptive dysphasia. His pupils were equal and reactive to light, extraocular movements were full, and visual fields were full to confrontation. There was a mild upper motor neuron right facial weakness, right hemiparesis that fluctuated in severity from mild to moderately severe, and a right pronator drift. His right plantar response was upgoing. Sensory examination and cerebellar function were normal.

\section{Imaging}

A computed tomogram (CT) scan was obtained, showing an old lacunar infarct in the left lentiform nucleus, an area of focal calcification in the left sylvian fissure, and early loss of grey- white matter differentiation in the lateral margin of the left insular cortex (Figure 1). Computed tomogram angiography revealed a densely calcified lesion in left distal internal carotid artery (ICA) extending into the proximal left middle cerebral artery (MCA) M1 segment, suggesting an embolus containing a mixture of calcium and acute blood clot (Figure 2). Calcified atheroma was noted along the aortic arch and in both carotid bifurcations, but there was no hemodynamically significant stenosis. The CT perfusion showed a large mismatch in the left MCA distribution, with a $10-20 \%$ reduction in blood flow, 40$50 \%$ reduction of blood volume, and moderate to severe delay in the mean transit time compared to the right side.
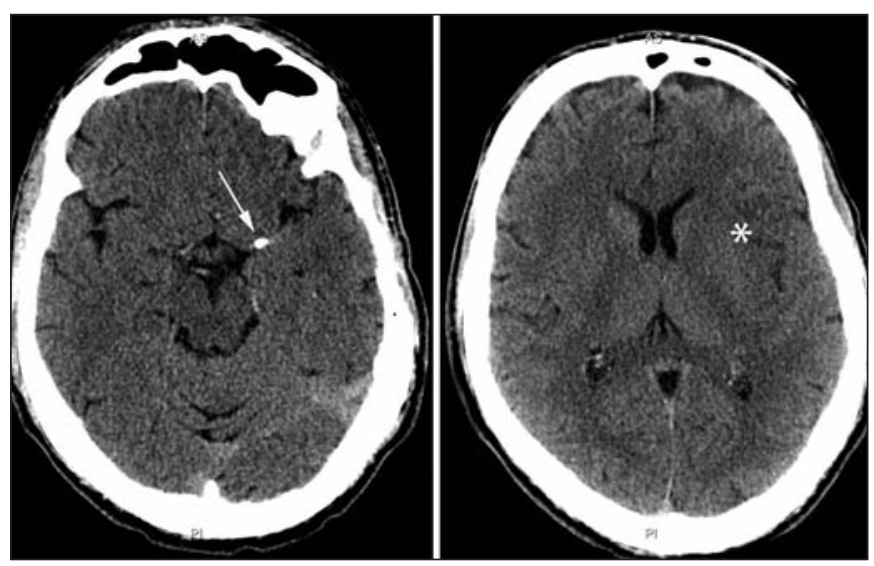

Figure 1: Axial non-contrast CT scan images obtained at the time of admission. Left: A focal area of calcification is present in the medial aspect of the left sylvian fissure (arrow). Right: Loss of grey-white differentiation in the left insula (asterisk).

From the Division of Neurosurgery, Department of Surgery, The University of British Columbia, Vancouver, British Columbia, Canada.

Received November 5, 2009. Final Revisions Submitted November 11, 2009 Correspondence to: Gary Redekop, Division of Neurosurgery, The University of British Columbia, 3100 - 910 West 10th Avenue, Vancouver, British Columbia, V5Z 4E3, Canada. 

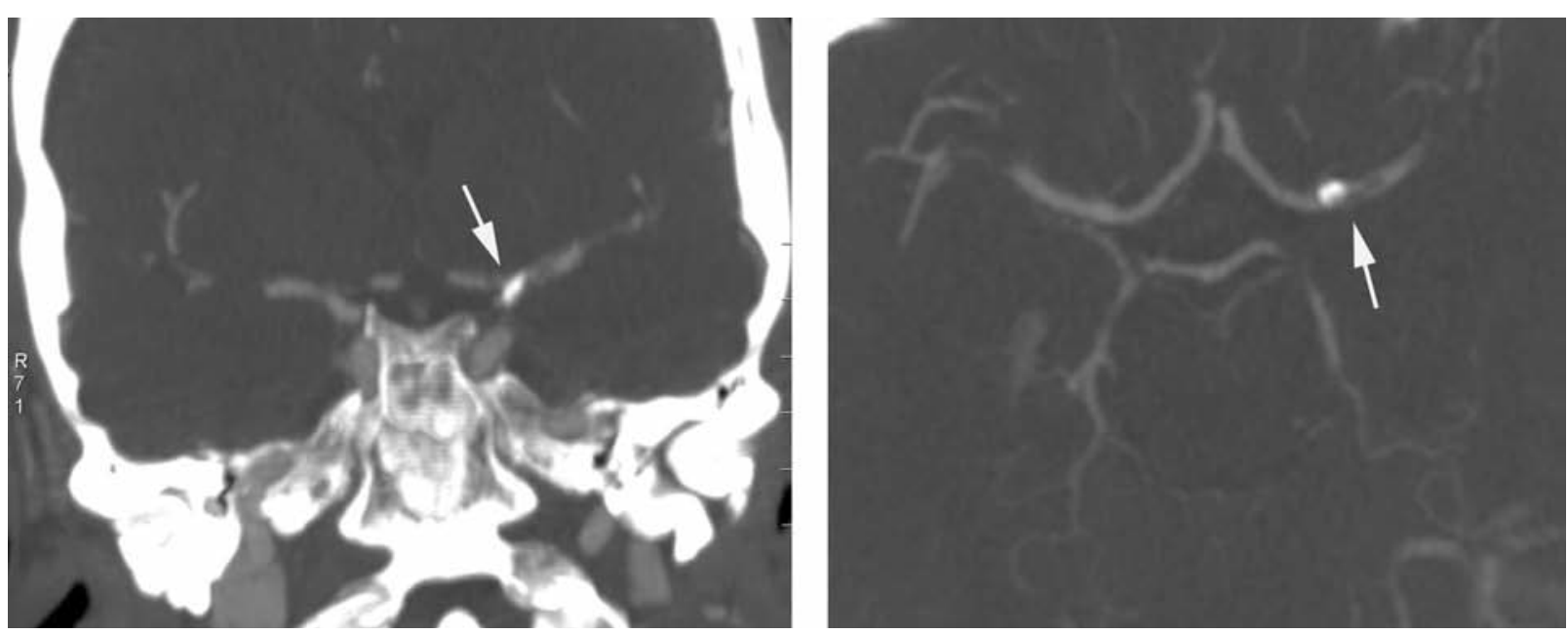

Figure 2: Coronal (left) and axial (right) CT angiogram images showing a partially calcified embolus extending from the distal left ICA into the proximal MCA (arrows).

\section{Endovascular Stroke Intervention}

The tissue in the territory of the left MCA was thought to be at risk of infarction and thrombolysis was felt to have a low probability of success given the calcified nature of the embolus. Therefore, an attempt at mechanical intravascular clot extraction under general endotracheal anesthesia was performed. Angiography showed delayed filling of the left ICA, due to a nearocclusive, large embolus in the distal left ICA and the proximal left M1 segment. However, contrast did flow around the embolus, resulting in partial filling of the left MCA territory, mainly confined to the Sylvian region. A right ICA injection was also performed, demonstrating normal circulation to the right hemisphere and excellent filling of the left anterior cerebral artery (ACA) territory via the anterior communicating artery (Figure 3).

The Merci Retriever ${ }^{\circledR}$ system was prepared and the microcatheter placed in the left M1 segment beyond the embolus over the guide wire. The clot retrieval device was deployed and two passes were made, without successful removal. A second Merci Retriever® system was prepared and two additional passes were made, again without success. The final angiogram showed multiple areas of mild spasm in the left internal carotid artery and complete occlusion of the left MCA M1 segment (Figure 4, left). The patient was taken to CT scan, which did not show significant change in comparison to the initial imaging, although perfusion studies were not obtained. No clinical examination was possible as the patient remained under anesthesia. At this time it was approximately five hours since the onset of the patient's symptoms.

\section{Surgical Procedure}

The patient was taken immediately to the operating room for MCA embolectomy. A left pterional craniotomy was performed and the sylvian fissure opened with sharp dissection, exposing the ICA and MCA. A firm and white partially calcified plaque embolus was seen occluding the distal ICA and extending onto the M1 segment of the MCA. Temporary clips were placed onto the ICA proximal to the occlusion, the A1 segment of the ACA, and the M1 segment of the MCA distal to the occlusion. A short arteriotomy incision was made in the proximal MCA over the embolus, and complete dissection and removal of the embolus was performed, followed by brief release of the proximal temporary clips on the internal carotid artery to flush out any residual debris. The arteriotomy was closed with a running 9-0 monofilament suture and the temporary clips removed. Pulsation
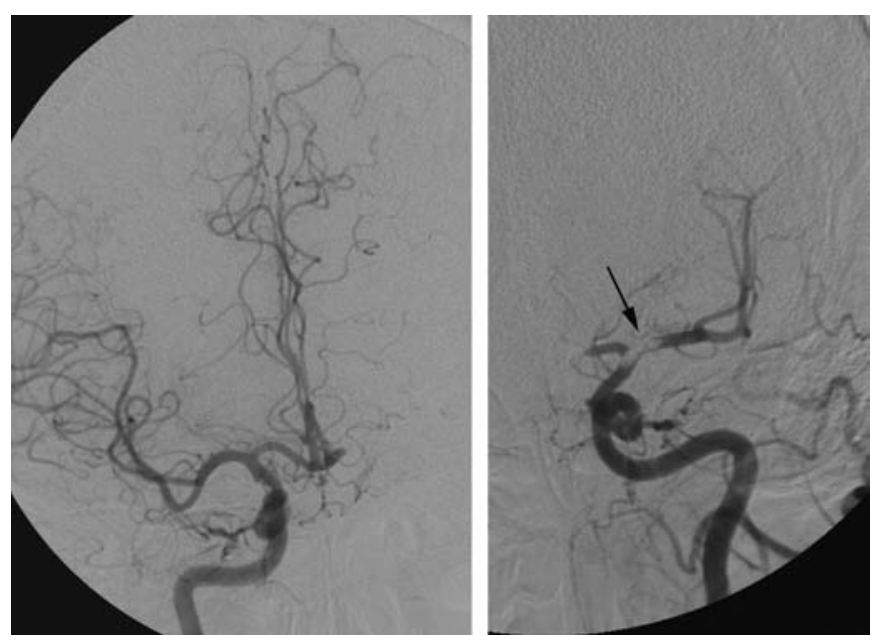

Figure 3: Left: Right carotid angiogram showing normal intracranial circulation, with spontaneous cross flow through the anterior communicating artery to the left ACA territory. Right: Left carotid angiogram showing a near-occlusive embolus extending from the distal left ICA into the proximal MCA (arrow), with limited antegrade perfusion of the left MCA territory and no filling of the distal ACA territory. 

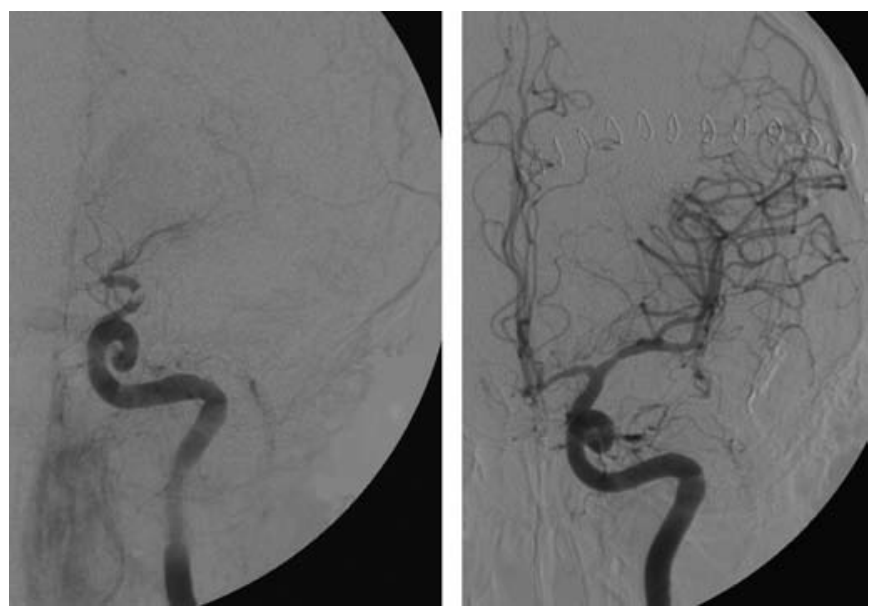

Figure 4: Left: Final angiogram obtained after failed attempt at mechanical embolus extraction, with complete occlusion of the left MCA. Right: Post-operative angiogram showing reconstitution of vessel patency, with normal left ACA and MCA circulation.

was immediately observed in the MCA and its branches, and confirmed with a micro doppler probe. The duration of temporary clipping was approximately 12 minutes, and normal cerebral perfusion was restored about 6.5 hours after symptom onset.

\section{Post-Operative Course}

Immediately following the surgery the patient awoke with moderate expressive dysphasia and right hemiparesis that improved significantly within 12 hours. A cerebral angiogram was performed two days post embolectomy, showing mild irregular narrowing of $\mathrm{A} 1$ and $\mathrm{M} 1$ of the left ACA and MCA, possibly related to vasospasm. No significant stenosis or thrombus or distal branch obstruction was seen (Figure 4, right). Light microscopy of the embolus showed fragments of focally calcified hypocellular tissue, displaying focal osseous metaplasia and fibrinoid degeneration consistent with arterial embolic material.

At the time of discharge from hospital five days postoperatively, the patient had mild slowing of fine movements in the right hand, and a mild dysphasia that was rapidly improving. When seen in the clinic six weeks post operatively, he complained of some headache and he and his wife noticed occasional slurring and word finding difficulty. His strength and co-ordination were normal and no focal deficit was found on neurological exam. A follow-up CT head scan was performed four months post operatively showing a small area of tissue loss in the left insula. Computed tomogram angiography showed that the left ICA and MCA were widely patent (Figure 5). Six months post operatively he returned to work full time. At the time of last follow-up, two and a half years post operatively, he reported occasional word finding difficulty, his previous headaches were gone, and he had no other neurological deficits.
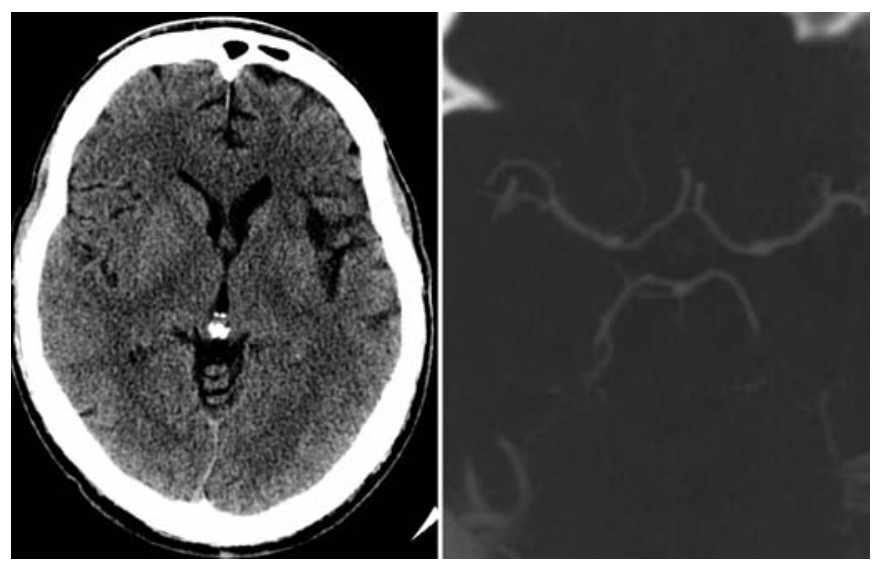

Figure 5: Post-operative imaging four months after surgery. Left: Axial CT scan showing a small infarct in the left insula. Right: CT angiogram showing patency of the left MCA.

\section{Discussion}

Current guidelines from the American Academy of Neurology recommend intravenous thrombolysis with rtPA for patients with acute ischemic stroke who can be treated within three hours of symptom onset, and who do not have contraindications to this therapy. ${ }^{1}$ The role of intra-arterial thrombolysis remains to be fully defined, but it is a treatment option for treatment of selected patients with ischemic stroke of less than six hours duration. ${ }^{1}$ Reliance on spontaneous lysis and resolution of MCA emboli has been rarely described, and patients who do not receive tPA have better clinical outcome if good collateral flow is demonstrated. ${ }^{5-7}$

Several non-pharmacologic endovascular treatments have been used for acute stroke caused by cerebral artery occlusion, such as mechanical disruption of the clot with guidewires or other devices, angioplasty and / or stenting, and mechanical extraction of the clot. ${ }^{8}$ The MERCI and Multi MERCI trials have reported the efficacy of endovascular clot retrieval using the Merci Retriever ${ }^{\circledR}$ in cases where intravenous rtPA is unavailable, contraindicated or inadequate for treatment of acute embolic stroke. $^{2-4}$ Although the use of the Merci Retriever ${ }^{\circledR}$ is FDA approved, its clinical utility is still being studied and its role in the emergency management of stroke remains to be established. ${ }^{1}$

Spontaneous calcific cerebral embolism is an uncommon cause of stroke, and there are no specific guidelines for treatment. Although the presence of calcified cerebral emboli is not a contraindication to intravenous thrombolysis, there have been some reports suggesting medical management is inadequate, and others where calcific cerebral emboli treated with thrombolysis resulted in downstream migration and fragmentation of the calcified cerebral embolus. ${ }^{9}$

Microsurgical embolectomy for acute middle cerebral artery occlusion was first reported by Welch in $1956 .{ }^{10}$ Since that time there have been several case reports and small series which suggest that embolectomy can be used safely in selected cases, 
although there is concern about the potential for conversion of a bland infarct into a hemorrhagic stroke, and uncertainty about the point at which it becomes futile due to irreversible neuronal injury..$^{11-17}$

In our case the high density mass seen on CT scan was suggestive of a highly calcified embolus that would be refractory to thrombolysis with rtPA, and intervention with endovascular mechanical clot retrieval was proposed as the most prudent treatment option. Unfortunately, the failed attempt produced an unexpected worsening of cerebral blood flow that required prompt reversal. Although normal antegrade flow was not restored for more than six hours after symptom onset, the patient made an excellent recovery and post-operative imaging demonstrated minimal evidence of permanent ischemic injury. The duration of complete MCA occlusion was not known with certainty, as the initial vascular imaging demonstrated some antegrade flow around the embolus, and there was at least modest antegrade flow and some leptomeningeal collateral perfusion prior to and during the attempted mechanical embolus extraction.

Emergency middle cerebral artery embolectomy for a calcific embolus with middle cerebral artery occlusion is an unproven but potentially beneficial therapy for patients with acute ischemia. Although applicable in only very selected situations, it remains a valuable adjunctive technique in the multi-disciplinary management of stroke.

\section{REFERENCES}

1. Adams H, del Zoppo G, Alberts M, Bhatt D, Brass L, Furlan A, et al. Guidelines for the early management of adults with ischemic stroke. Stroke. 2007;38:1655-711.

2. Gobin YP, Starkman S, Duckwiler GR, Grobelny T, Kidwell CS, Jahan R, et al. MERCI 1: a phase 1 study of mechanical embolus removal in cerebral ischemia. Stroke. 2004;35:2848-54.

3. Smith WS. Safety of mechanical thrombectomy and intravenous tissue plasminogen activator in acute ischemic stroke. Results of the Multi Mechanical Embolus Removal in Cerebral Ischemia (MERCI) Trial, Part I. Am J Neuroradiol. 2006;27:1177-82.

4. Smith WS, Sung G, Starkman S, Saver JL, Kidwell CS, Gobin YP et al. Safety and efficacy of mechanical embolectomy in acute ischemic stroke: results of the MERCI trial. Stroke. 2005;36: $1432-8$.

5. Dalai PM. Cerebral embolism. Angiographic observation on spontaneous clot lysis. Lancet. 1965;1:61-4.

6. Fieschi C, Bozzao L. Transient embolic occlusion of the MCA cerebral artery and internal carotid arteries in cerebral apoplexy. J Neurol Neurosurg Psychiatry. 1969;32:236-40.

7. Bozzao L, Fantozzi LM, Bastianello S, Bozzao A, Fieschi C. Early collateral blood supply and late parenchymal brain damage in patients with middle cerebral artery occlusion. Stroke. 1989;20: $735-40$

8. Schumacher HC, Meyers PM, Yavagal DR, Harel NY, Elkind MS, Mohr JP, et al. Endovascular mechanical thrombectomy of an occluded superior division branch of the left MCA for acute cardioembolic stroke. Cardiovasc Intervent Radiol. 2003;26: 305-8.

9. Kavanagh EC, Fenton DM, Heran MK, Lapointe JS, Nugent RA, Graeb DA. Calcified cerebral emboli. AJNR Am J Neuroradiol. 2006;27:1996-9.

10. Welch K. Excision of occlusive lesions of the middle cerebral artery. J Neurosurg. 1956;13:73-80.

11. Garrido E, Stein BM. Middle cerebral artery embolectomy. Case report. J Neurosurg. 1976;44:517-21.

12. Horiuchi T, Nitta J, Sakai K, Tanaka Y, Hongo K. Emergency embolectomy for treatment of acute middle cerebral artery occlusion. J Neurosurg. 2007;106:257-62.
13. Kakinuma K, Ezuka I, Takai N, Yamamoto K, Sasaki O. The simple indicator for revascularization of acute middle cerebral artery occlusion using angiogram and ultra-early embolectomy. Surg Neurol. 1999;51:332-41.

14. Khodadad G. Middle cerebral artery embolectomy and prolonged widespread vasospasm. Stroke. 1973;4:446-50.

15. Linskey ME, Sekhar LN, Hecht ST. Emergency embolectomy for embolic occlusion of the middle cerebral artery after internal carotid artery balloon test occlusion: case report. J Neurosurg. 1992;77:134-8.

16. Meyer FB, Piepgras DG, Sundt TM Jr, Yanagihara T. Emergency embolectomy for acute occlusion of the middle cerebral artery. J Neurosurg. 1985;62:639-47.

17. Sakai K, Nitta J, Horiuchi T, Ogiwara T, Kobayashi S, Tanaka Y, et al. Emergency revascularization for acute main-trunk occlusion in the anterior circulation. Neurosurg Rev. 2008;31:69-76. 